\title{
The effects of surge motion on floating horizontal axis tidal turbines
}

\author{
Mohamad H. B. Osman, Richard H. J. Willden, and Christopher R. Vogel
}

\begin{abstract}
Wave induced motions due to actual sea state conditions will impact the performance of floating horizontal axis tidal turbine systems. This paper presents the results from numerical simulations of a 3-bladed horizontal axis tidal turbine oscillating in surge motion in a moving reference frame. The optimum tip-speed ratio, $\lambda$ $=4.4$ and $k-\omega S S T$ turbulence model were used in the present study. The Navier-Stokes equation was modified by adding an inertial term to the equation and the Dirichlet boundary condition was also modified in order to simulate in the moving reference frame. The surge oscillations were parameterised in terms of the ratio of surge amplitude to rotor radius, $A^{*}$, and the ratio of oscillation frequency to the rotational frequency of the rotor, $\omega^{*}$. A series of tests were conducted to study the effect of each parameter on the hydrodynamic performance of the tidal turbine. The results show that stall can occur on the blade when the velocity relative to the rotor is sufficiently high. In certain cases, negative thrust and power coefficients were observed when the velocity relative to the rotor is low. The fluctuation in blade loading increases together with the amplitude and frequency of oscillation, which will contribute to the fatigue of the rotor.
\end{abstract}

Keywords-Floating tidal turbine, non-inertial reference frame, OpenFOAM

\section{INTRODUCTION}

$\mathrm{T}$ HE demand for energy increases every year with the continued growth of economies around the world. Many countries have begun developing technologies to harness clean and renewable energy resources, which includes tidal current energy [1], [2], [3]. In 2016, Scottrenewables deployed a floating tidal turbine at the European Marine Energy Centre (EMEC), and the device has shown commercial potential in the field of tidal energy. Floating tidal turbines may be more suitable for deep water (depth $>30 \mathrm{~m}$ ) operations rather than shallow water, as the flow speed in a channel is highest near the

Manuscript received 16 March; accepted 26 March; published 8 Sept, 2020. This is an open access article distributed under the terms of the Creative Commons Attribution 4.0 licence (CC $\quad$ BY https://creativecommons.org/licenses/by/4.0/). This article has been subject to single-blind peer review by a minimum of two reviewers. This work was supported in part by Majlis Amanah Rakyat Malaysia (MARA) and Universiti Teknologi Malaysia (UTM). M. H. B. Osman, R. H. J. Willden and C. R. Vogel are with the Department of Engineering Science, University of Oxford, Parks Road, Oxford OX1 3PJ, UK (e-mail: mohamad.binosman@eng.ox.ac.uk). Digital Object Identifier https://doi.org/10.36688/imej.3.45-54 water surface due to the vertical shear profile [4]. Thus, more power is available to turbines positioned higher in the water column, due to the higher flow speeds. However, the turbines will also experience higher loads. Furthermore, flow unsteadiness due, for example, to passing waves and floating support platform (or buoy) motion will also have an impact on turbine performance and structural requirements.

Whilst most research to date revolves around fixed tidal turbines, relatively little has been conducted on floating tidal turbines. References [1], [2], [3] investigated the hydrodynamic performance of a tidal turbine in yaw, surge, and roll motions, respectively. These studies used computational fluid dynamics (CFD) models to simulate the floating tidal turbine. The surge and roll motion studies used a two-bladed horizontal axis tidal turbine (HATT) with a diameter of $0.7 \mathrm{~m}$ while the yaw motion study considered a vertical axis tidal turbine (VATT). The motion of the turbines was implemented by using the mesh morphing (deforming mesh) method, in their respective motion paths, to simulate the effects of floating platform motion on the device. It was shown in each case that the oscillations increase the fatigue loads on the turbine due to the resulting oscillating loads. The added mass (inertial) and damping coefficients associated with these motions were also derived from these studies. Surge motion was shown to have the greatest impact on turbine loading, compared to roll and yaw motion.

The present study focuses on the hydrodynamic performance of a turbine oscillating in surge motion. The turbine was prescribed to oscillate in 1 degree of freedom (DoF) over a range of oscillation amplitudes and frequencies. The present study uses the moving reference frame method to reduce the computational cost and maintain mesh quality, in comparison to the mesh morphing methods used in previous studies.

\section{COMPUTATIONAL MODEL}

The present study investigates the hydrodynamic performance of a turbine oscillating in surge motion. A 3bladed HATT, with a diameter of $20 \mathrm{~m}$ and designed for operation in blocked flows, was chosen for the present study because this type of turbine is widely proposed for use in commercial applications. A moving reference frame was used to simulate the surge motion of the turbine. The flow was described by the unsteady 


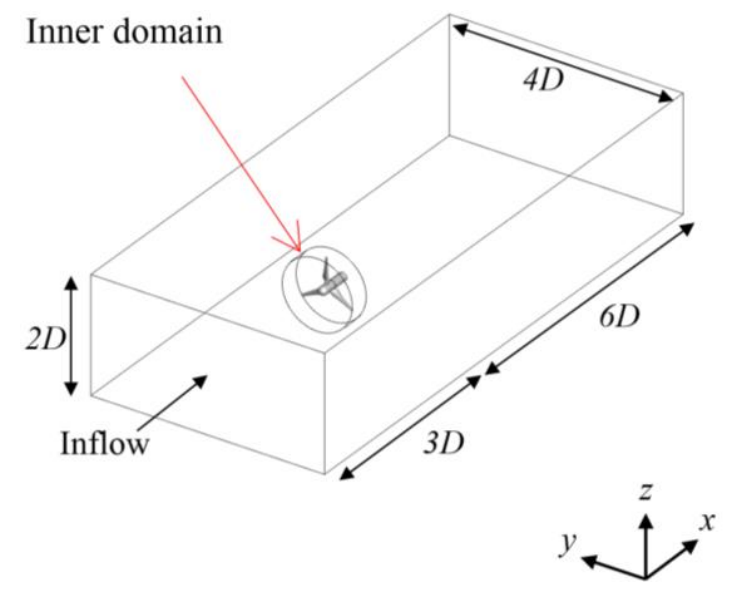

Fig. 1. Diagram of the computational domain showing the outer and inner domain regions, where the rotor and the coin-shaped domain surrounding it form the inner domain and the rest is the outer domain. The flow is in the positive $\mathrm{x}$-direction and rotor rotation is in the clockwise direction (viewed from the flow direction).

Reynolds-Averaged Navier-Stokes (RANS) equations, with the $k-\omega$ SST model as described by Menter [5] used for turbulence modelling.

\section{A. Computational Domain}

The rotor used in this study was developed by [6] as a modification of the rotor design by [7]. The rotor uses the RIS $\emptyset$-A1-24 aerofoil section along the blade span. Reference [7] demonstrated that this rotor design gives better performance in blocked operating conditions compared to rotors designed for unblocked flow. The rotor diameter, $D$, is $20 \mathrm{~m}$ and the nacelle diameter is $0.15 D$. The rotor dimensions were selected to be representative of a full-scale tidal turbine. Further details on how the rotor mesh was designed can be found in [6].

Following [8], the rectangular outer domain consists of a channel with a width of $4 D$ and a depth of $2 D$. The rotor is placed at the centre of the channel. The rotor is located $3 D$ downstream from the inlet and $6 D$ upstream from the outlet of the domain. This is to ensure that the flow will be stabilized before reaching the rotor and to stabilize the wake in the downstream region. The overall computational domain is shown in Fig. 1. The blockage ratio, $B$ (ratio of the rotor swept area, $A_{D}$, to the area of channel cross section) for the domain is 0.0982 . The mesh for the outer domain was constructed using an O-grid blocking strategy. Note that for the present study, only the outer domain mesh was constructed while the inner domain mesh provided directly from [6].

The inner domain is a thin 'coin' region, $0.25 D$ in length that includes the rotating turbine rotor. This contains 2.6 million elements in a block-structured grid. Table I shows a summary of the mesh sensitivity study for coarse, medium, and fine meshes of the outer domain. A growth rate of 1.2 away from the rotor plane was used for each of the meshes up to a distance of $1.3 D$ upstream and downstream of the front and back of the inner
TABLE I

SUMMARY OF MESH SENSITIVITY STUDY FOR THE OUTER DOMAIN

\begin{tabular}{lllllll}
\hline & $\begin{array}{l}\text { No. of } \\
\text { cells } \\
(\text { million }\end{array}$ & $\begin{array}{l}\text { Minim } \\
\text { um cell } \\
\text { size }\end{array}$ & $C_{P}$ & $C_{T}$ & $\begin{array}{l}\Delta C_{P} \\
(\%)\end{array}$ & $\begin{array}{l}\Delta C_{T} \\
(\%)\end{array}$ \\
& (x 10-4) & & & & & \\
\hline Coarse & 0.25 & 6.27 & 0.519 & 1.018 & 0.03 & 0.38 \\
Medium & 0.52 & 1.82 & 0.519 & 1.016 & 0.03 & 0.27 \\
Fine & 1.10 & 0.52 & 0.519 & 1.014 & - & - \\
\hline
\end{tabular}

domain, respectively. Further upstream and downstream of this, the meshes were extended without any further growth in element size.

The outer and inner domains were connected using Arbitrary Mesh Interfaces (AMIs). This 'separate domain' approach was taken to enable the construction of a finer mesh around the rotor for the inner domain without continuing the mesh into the outer domain. This helps to reduce the total cell count of the entire computational domain and eases the meshing process.

\section{B. Turbulence Model}

A comparison study between Large Eddy Simulation (LES) and RANS models was conducted by [9] on the effects of turbulent flow and loading on a HATT. It was demonstrated that the computational cost for LES modelling is much higher than a comparable RANS model while showing only a small difference between the turbine performance predicted by the two modeling approaches. Therefore, for the present study a RANS model will be used. The $k-\omega$ SST model, a two equation turbulence model which combines the $k-\omega$ model and $k-\varepsilon$ model where the former is applied in the near wall region and the latter is applied in the outer region and in a free shear flow [5], was chosen for the present study.

\section{Non-Inertial Frame of Reference}

One way to simulate an oscillating tidal turbine rotor is by using a mesh morphing (deforming mesh) method in which the rotor is oscillated back and forth while rotating around a fixed point (for surge motion), inside the domain. By doing so, the mesh cells are stretched and compressed along the outer domain where the oscillation takes place. In addition to the long computational time associated with mesh deformation, this approach can cause a high level of skewness inside the domain which may lead to poor mesh quality [10], [11]. For the present study, the mesh quality of the computational domain is of primary importance and therefore the mesh morphing strategy was considered to not be suitable.

Another approach to model this problem is to simulate the flow in a frame of reference attached to the (nonrotating) turbine nacelle, i.e. in a non-inertial moving coordinate system. The Navier-Stokes equations need to be modified following the method presented by [12] to model surge motion in a moving reference frame. Although the flow around the rotor in both frames of reference is kinematically the same, they differ 


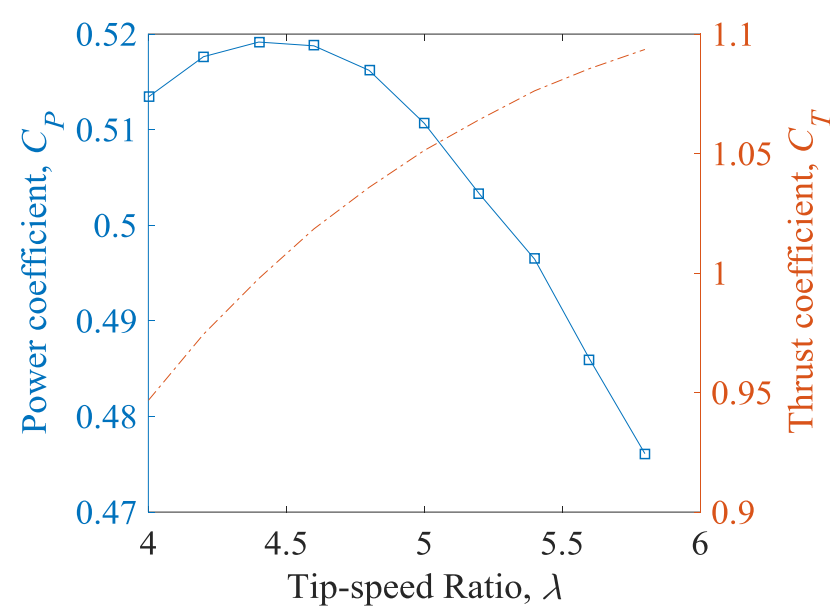

Fig. 2. Power and thrust coefficient variation over a range of tipspeed ratios. The optimum tip-speed ratio for the rotor in the present study is $\lambda=4.4$.

dynamically in the acceleration (inertial) term [13], [14]. By adopting a moving frame of reference, the mesh remains the same throughout the simulation, maintaining the mesh quality of the computational domain. Note that the inner mesh containing the rotor still rotates about the $x$-axis of the moving reference frame in order to simulate rotor rotation.

The surging motion of the floating turbine is modelled in the non-inertial (moving) reference frame, where the coordinate system is fixed at the rotor. The Navier-Stokes equations are modified following the generalised method presented by $\mathrm{Li}$ et al. [12] to account for the motion of a body as follows

$$
\nabla \cdot U=0
$$

$$
\begin{gathered}
\partial U / \partial t+U(\nabla \cdot U)=-\nabla p+v\left(\nabla^{2} \cdot U\right)+B \\
B=\dot{\omega} \times X-\ddot{x}+\omega \times(\omega \times X)+2 \omega \times U_{A}
\end{gathered}
$$

where $U$ and $p$ are the velocity vector and pressure in the moving reference frame, $x$ is the translational displacement of the moving object in the inertial frame, $\omega$ is the angular frequency of the rotating object, $X$ is the position vector relative to the moving reference frame, and $U_{A}$ is the object's velocity relative to the moving reference frame. The (') and (") represent the first and second time derivative (velocity and acceleration), respectively. The terms in $(2 b)$, from left to right, are: acceleration due to the change in angular velocity, translational acceleration of the moving body, centrifugal acceleration, and Coriolis acceleration. For the present study, since the rotor is oscillating only in surge motion, only the second term was added into the Navier-Stokes equation. The corresponding far-field velocity Dirichlet boundary condition then becomes

$$
U(t)=U_{a p}(t)=U_{\infty}-\dot{x}(t)
$$

where $U_{\infty}$ is the unperturbed inflow velocity in the farfield, $U_{a p}(t)$ is the apparent velocity seen by the turbine, and $\dot{x}(t)$ is the velocity of the moving turbine in surge motion. Further details about the change in frame of reference can be found in [12]. Symmetry boundary conditions were applied to the top, bottom and lateral boundaries.

A uniform inflow profile with a velocity of $2 \mathrm{~m} / \mathrm{s}$ was used. Simulation of a floating offshore tidal turbine in a sheared flow profile will be considered in future work. A modified solver was compiled in OpenFOAM v2.3.x to add the acceleration term presented in (1). An additional boundary condition library was used to modify the Dirichlet boundary condition [11]. The fluctuating inflow velocity to represent rotor surge oscillation in the moving reference frame was modelled as a simple sinusoid:

$$
U_{a p}(t)=U_{\infty}-A_{0} \omega_{0} \cos \left(\omega_{0} t\right)
$$

where $U_{a p}$ is the velocity relative to the rotor (apparent velocity seen by the turbine), $A_{0}$ and $\omega_{0}$ are the amplitude and frequency of the surge oscillation, respectively. The second term of the equation is the velocity of the oscillating turbine where the displacement is given by $x(t)=A_{0} \sin \left(\omega_{0} t\right)$.

\section{EFFECT OF SURGE MOTION ON TURBINE PERFORMANCE}

This paper investigates the effects of two surge oscillation parameters; surge amplitude and surge frequency, on tidal turbine performance. Firstly, the effects of surge amplitude on turbine performance were investigated by varying the surge amplitude ratio $A^{*}=$ $A_{0} / R$, where $R$ is the rotor radius. Secondly, the effects of surge frequency were investigated by varying the frequency ratio $\omega^{*}=\omega_{0} / \omega_{r}$, where $\omega_{r}$ is the frequency of rotation of the rotor.

\section{A. Optimum Tip-Speed Ratio}

In order to determine the optimum tip speed ratio, $\lambda$, of the turbine, a series of steady flow multiple reference frame (MRF) simulations were carried out across a range of $\lambda$ from 4.0 to 5.8. In the MRF simulation the turbine is held stationary in a cylindrical domain (with the same overall blockage) and the inflow is rotated as it approaches the stationary rotor. MRF simulations were used to determine the optimum $\lambda$ of the turbine as this method is computationally cheaper than the sliding mesh (transient) method. The surge oscillation simulations used the sliding mesh method in order to simulate rotor rotation.

The thrust and power coefficients were defined as follows:

$$
C_{T}(t)=\frac{T(t)}{\frac{1}{2} \rho U_{\infty}^{2} A_{D}}
$$




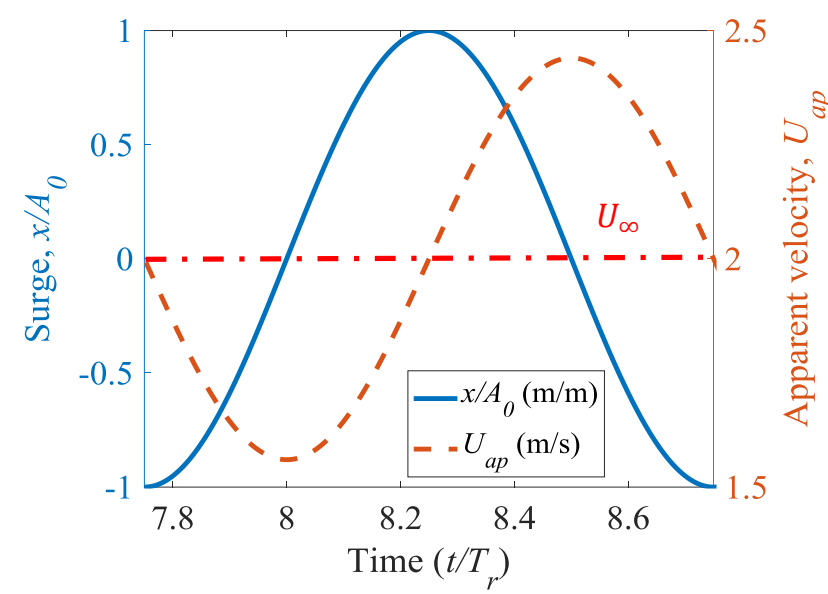

Fig. 3. Normalized surge and apparent velocity time histories for a turbine surging at surge amplitude and frequency of $A_{0}=0.5 \mathrm{~m}$ and $\omega_{0}=0.88 \mathrm{rad} / \mathrm{s}$, respectively. The inflow velocity $U_{\infty}=2 \mathrm{~m} / \mathrm{s}$.

$$
C_{P}(t)=\frac{Q(t) \omega_{r}}{\frac{1}{2} \rho U_{\infty}^{3} A_{D}}
$$

where $T(t)$ and $Q(t)$ are the thrust and torque, respectively, as a function of time, turbine swept area $A_{D}=\pi R^{2}$ and $\rho=1000 \mathrm{~kg} / \mathrm{m}^{3}$ is the fluid density. Fig. 2 shows the variation in power and thrust coefficients over a range of tip-speed ratios, $\lambda$. It was found that the optimum $\lambda$ for the turbine in the present study is $\lambda=4.4$.

\section{B. Comparison Between Fixed and Surging Turbines}

Fig. 3 shows the relationship between surge motion and the apparent velocity $U_{a p}$ for a surging turbine oscillating with amplitude $A_{0}=0.5 \mathrm{~m}$ and frequency $\omega_{0}=$ $0.88 \mathrm{rad} / \mathrm{s}$ (i.e. $A^{*}=0.05$ and $\omega^{*}=1.00$ ) rotating at a tip speed ratio $\lambda=4.4$. Note that this case will be considered for further analysis as a representative case. $T_{r}$ is the time for one rotor revolution at the optimum tip speed ratio $\lambda$ $=4.4$. Surge motion in the duwrstream direction is considered to be positive, whilst the negative direction corresponds to the rotor moving in the upstream direction, into the approaching flow. $U_{a p}$ is $90^{\circ}$ out of phase with the surge motion, and is maximum when the rotor advances into the oncoming flow, and is minimum when it moves in the downstream direction.

Fig. 4 shows the time histories of $C_{P}$ and $C_{T}$ for a fixed turbine and a surging turbine. As the rotor oscillates in the upstream direction, we observe an increase in $C_{P}$ and $C_{T}$ compared to the fixed turbine, which is partially due to the increase in $U_{a p}$. Conversely, $C_{P}$ and $C_{T}$ drop below the fixed turbine case due to the decrease in $U_{a p}$ when the rotor oscillates in the downstream direction. The oscillations in $C_{P}$ and $C_{T}$ are largely in phase with $U_{a p}$ as the power and thrust are proportional to the velocity cubed and velocity squared, respectively.

We can classify the surge motion into four stages as shown in Fig. 5. Stage 1 and stage 2 comprise the period in which the turbine accelerates and decelerates in the downstream direction respectively, whereas stages 3 and 4 are where the turbine accelerates and decelerates in the
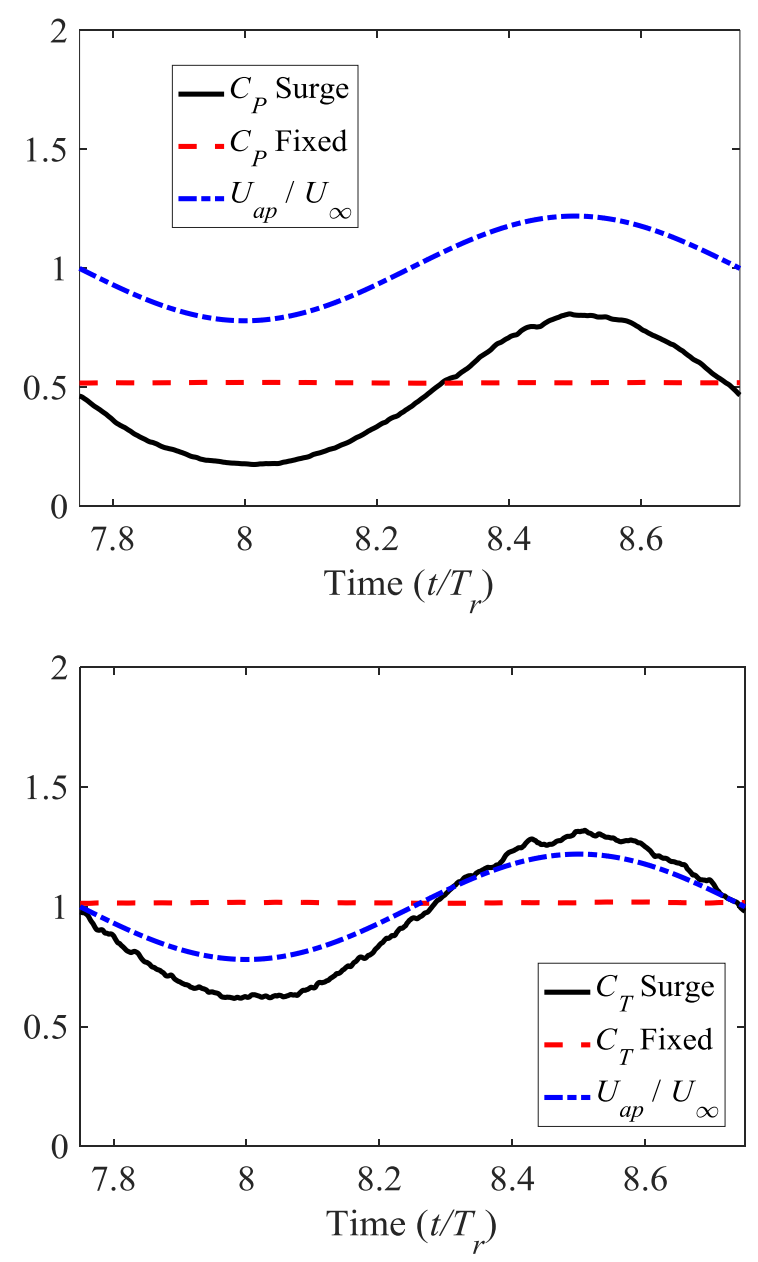

Fig. 4. Time histories of $C_{P}$ (top) and $C_{T}$ (bottom) for a fixed turbine and a surging turbine oscillating at $A_{0}=0.5 \mathrm{~m}$ and $\omega_{0}=0.88$ $\mathrm{rad} / \mathrm{s}$. The turbines rotate at a constant $\lambda=4$.4. The time history of $U_{a p}$ normalized by $U_{\infty}$ is also plotted.

upstream direction. As the power and thrust vary over time, it is informative to consider the stages in which the rotor produces the maximum and minimum power and thrust. For convenience, we define the power output ratio, $P_{r}$, and thrust ratio, $T_{r}$, for each stage as follows:

$$
\begin{gathered}
P_{r}=\frac{\bar{C}_{P \text { surge }}}{\bar{C}_{P \text { fixed }}} \\
T_{r}=\frac{\bar{C}_{T \text { surge }}}{\bar{C}_{T \text { fixed }}}
\end{gathered}
$$

where $\bar{C}_{P \text { surge }}$ and $\bar{C}_{T \text { surge }}$ are the time-averaged values of $C_{P}$ and $C_{T}$, respectively, during each motion stage of the surging turbine. $\bar{C}_{P \text { fixed }}$ and $\bar{C}_{T \text { fixed }}$ are the average values of normalized power and thrust of the fixed turbine (which are equal to the power and thrust coefficients, respectively).

Fig. 6 shows the $P_{r}$ and $T_{r}$ at each stage. The minimum and maximum $P_{r}$ and $T_{r}$ occurs at stages 2 and 4 respectively. When the turbine moves in the upstream direction (stage 3 and stage 4), $U_{a p}$ is greater than the mean velocity, leading to higher rotor power and thrust. 


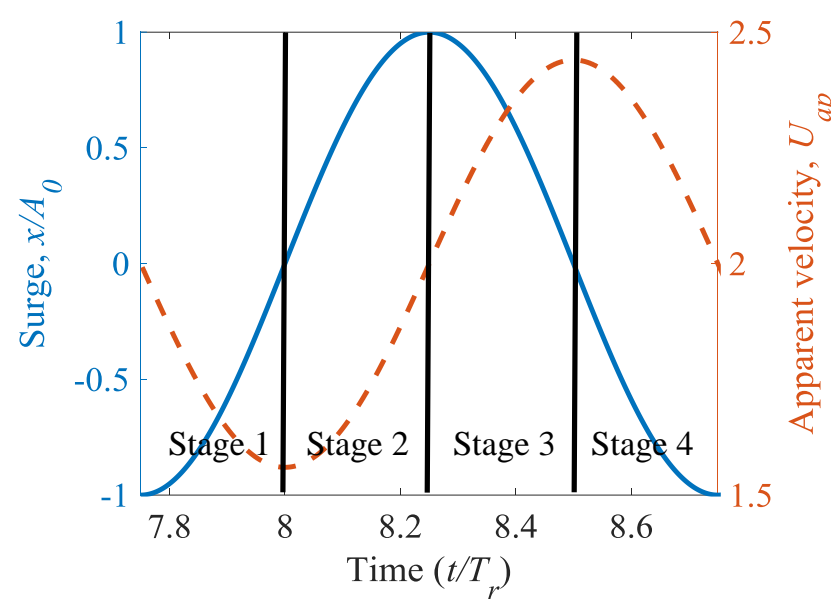

Fig. 5. Stages of the turbine in one cycle of oscillation at $A_{0}=0.5 \mathrm{~m}$ and $\omega_{0}=0.88 \mathrm{rad} / \mathrm{s}$.
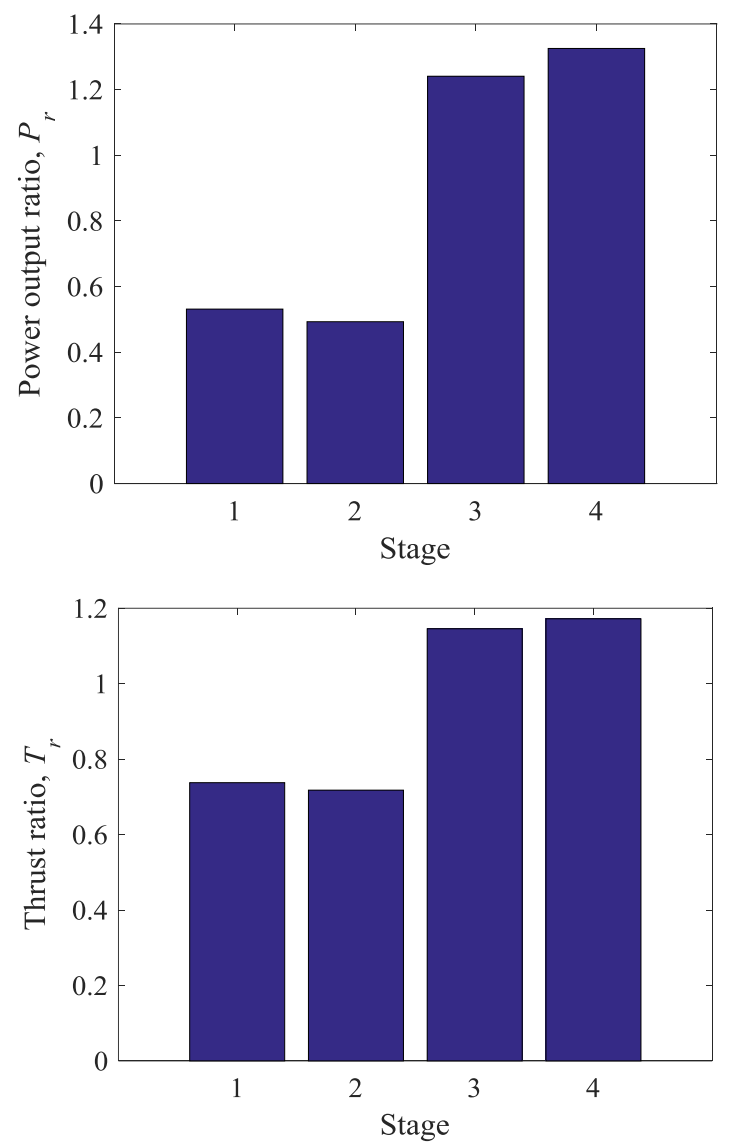

Fig. 6. Power output ratio and thrust ratio of the four stages of the surging turbine oscillating at $A_{0}=0.5 \mathrm{~m}$ and $\omega_{0}=0.88 \mathrm{rad} / \mathrm{s}$.

When the turbine moves in the downstream direction (stages 1 and 2), the apparent velocity decreases resulting in lower rotor power and thrust. Comparing stages 1 and 2 , and stages 3 and 4 , although the average $U_{a p}$ is the same for both stages, $P_{r}$ and $T_{r}$ show slightly different values indicating same dynamic effects that cannot be simply accounted for in a quasi-steady. The values of $P_{r}$ and $T_{r}$ in each stage consequently depend on the flow conditions and wake structure developed in previous stages.
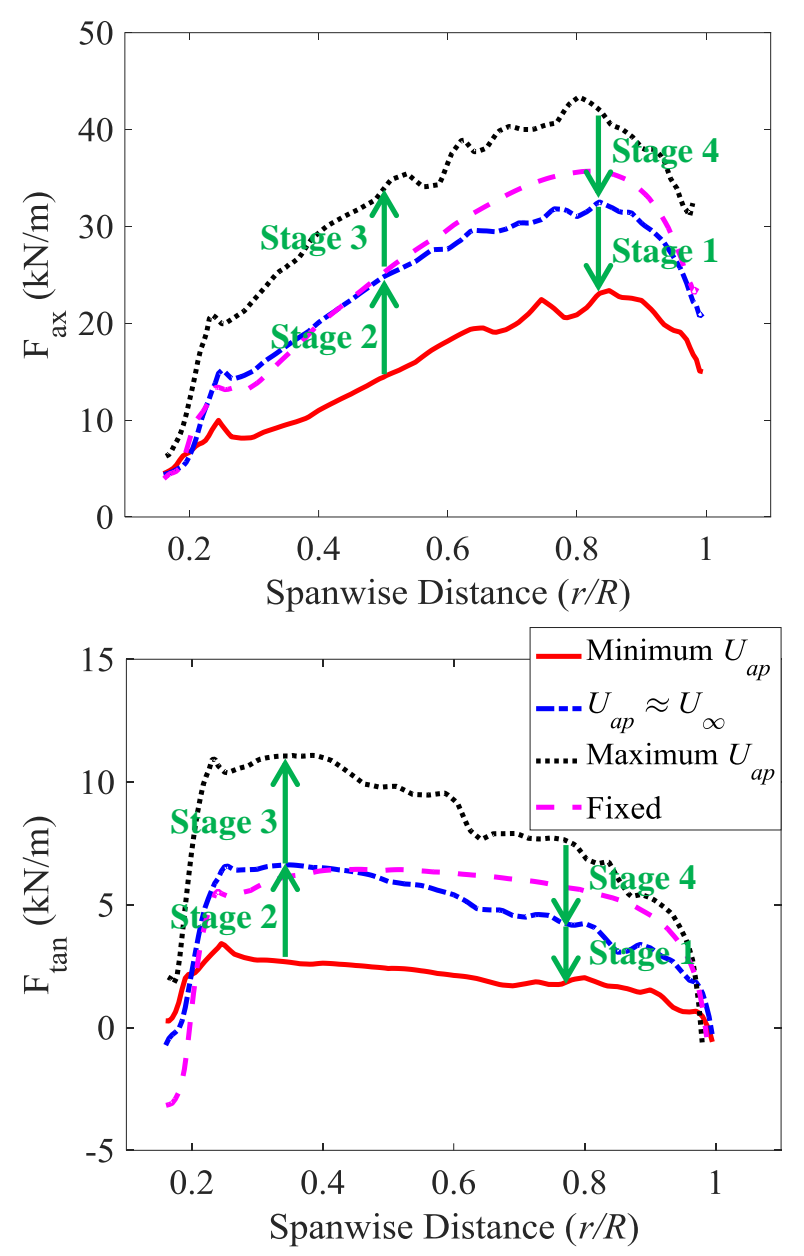

Fig. 7. Axial (top) and tangential (bottom) force profiles along the blade for fixed and surging turbines at three time steps which correspond to the minimum $U_{a p}, U_{a p} \approx U_{\infty}$, and maximum $U_{a p}$.

Fig. 7 shows the axial and tangential forces along the blade for the surging and fixed turbine cases. Loading on the blade increases and decreases with $U_{a p}$ for the surging turbine case. Three points in the cycle are considered; when $x / A_{0}=0$ and the turbine is advancing, leading to maximum $U_{a p}$, when the turbine is at $x / A_{0} \approx-1$ and $U_{a p} \approx U_{\infty}$, and when $x / A_{0}=0$ and the turbine is retreating, leading to minimum $U_{a p}$. We observe that the spanwise force profiles of the surging turbine when $U_{a p} \approx$ $U_{\infty}$ are not the same as those for the fixed turbine, demonstrating that there are dynamic effects associated with the acceleration and deceleration of the surging turbine. Such effects are not apparent when using a quasisteady simulation methods such as Blade Element Momentum (BEM) theory. References [15], [16] make comparisons between BEM and CFD models, showing that one reason BEM under predicts the power and thrust variations is because the annular independence assumption in BEM does not all consideration of radial flow along the blade. Furthermore, wake expansion and contraction are not modelled in BEM, which renders the model unable to capture the unsteady wake dynamics effects, reducing accuracy. 


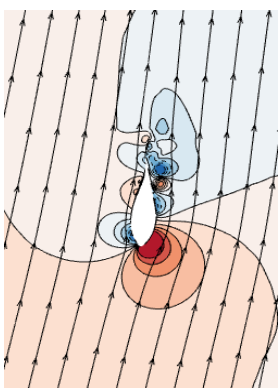

(a) Axial velocity

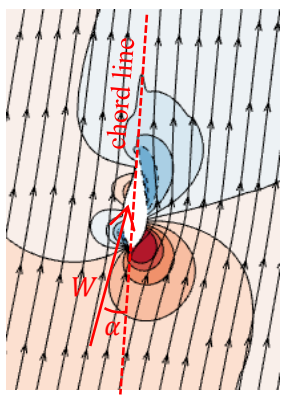

(c) Axial velocity

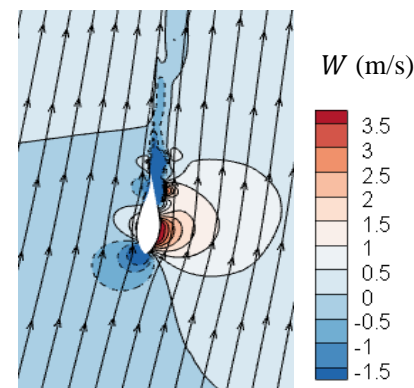

(b) Tangential velocity

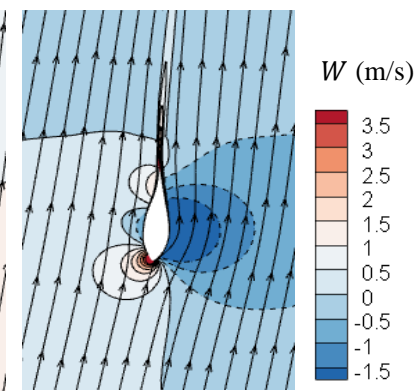

(d) Tangential velocity

Fig. 8. Contour plots of axial and tangential velocities relative to the blade at $r / R=0.8$ for a surging ( $\mathrm{a}$ and b) and fixed (c and d) and turbine at $t / T_{R}=8.25$, where $U_{a p} \approx U_{\infty}$. Dashed lines indicate negative contour values. Streamlines of the resultant velocity $W$ are superimposed. $\alpha$ is the instantaneous angle of attack.

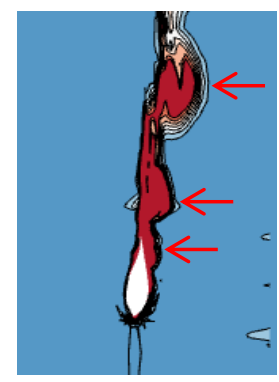

(a) $t / T_{r}=8.25$ (surging)

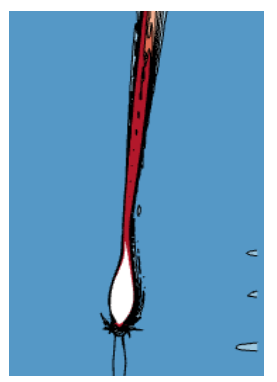

(b) Fixed

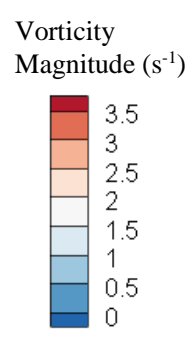

Fig. 9. Contour plots of vorticity magnitude at $r / R=0.8$ for surging (a) and fixed (b) turbines at $t / T_{r}=8.25$, where $U_{a p} \approx U_{\infty}$.

Comparison of the axial and tangential relative velocities between the surging turbine at $t / T_{r}=8.25$ $\left(U_{a p} \approx U_{\infty}\right)$ and the fixed turbine case are presented in Fig. 8 for a blade cross-section at $r / R=0.8$, as this is the location at which the blade forces see the most significant change. The streamlines of the resultant velocity relative to the blade, $W$, are superimposed onto the contour plots. For the surging turbine case, regions of swirling and reversed flow occur from the trailing edge to the leading edge on the suction side of the blade. These shed vortices occur due to the unsteadiness and time dependant blade loading during surge motion.

In Fig. 9, a vortex street is observed behind the blade section in the surging turbine case. The vortex street occurs due to vortex pumping, marked by the red arrows, as a result of time-varying blade loading. Vortex pumping does not occur for the fixed turbine case, and only trailing vortices convect downstream of the blade.

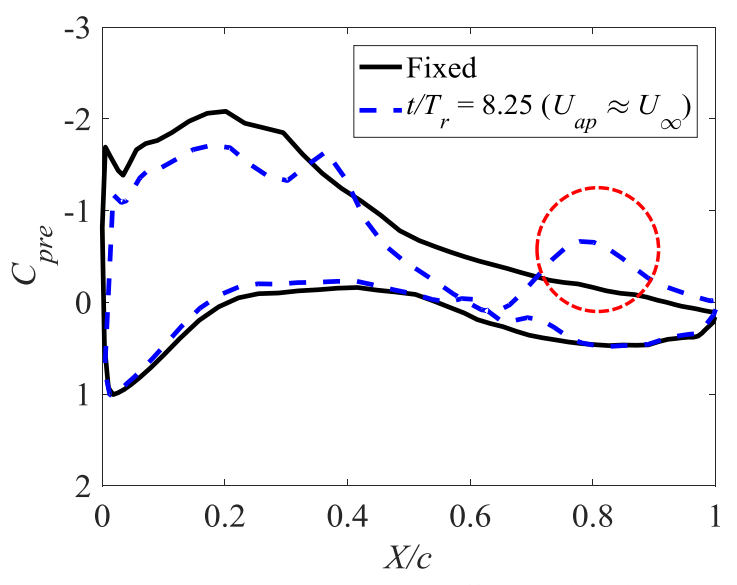

Fig. 10. Instantaneous pressure coefficient distribution at blade cross-section $r / R=0.8$ for fixed (solid) and surging (dashed) turbines at $t / T_{r}=8.25$, where $U_{a p} \approx U_{\infty}$.

As shown in Fig. 10, there is little difference in the pressure distribution on the pressure side of the blade in the fixed and surging cases. However, there are significant differences on the suction side of the blade. The pressure variation highlighted in Fig. 10 is a result of vortex shedding occurring towards the rear of the blade. Further vorticity is shed just downstream of the suction side, and also towards the trailing edge on the pressure surface.

\section{Effect of Surge Amplitude on Turbine Performance}

Three different surge amplitude ratios, $A^{*}=0.05,0.10$, 0.15 were investigated. Throughout this investigation $\omega^{*}$ $=1.0$ and $\lambda=4.4$ are held constant. The values of $A^{*}$ chosen for this study correspond to surge oscillation amplitudes of between $0.5 \mathrm{~m}$ to $1.5 \mathrm{~m}$ for a $20 \mathrm{~m}$ diameter rotor.

Fig. 11 shows the time variation of $C_{P}$ and $C_{T}$ for the three different surge amplitudes. Only data from the last two cycles were used for the analysis to ensure statistically converged results. The amplitude of $C_{P}$ and $C_{T}$ fluctuations are observed to increase as surge amplitude increases. There are asymmetries at the peak of each cycle for the cases $A^{*}=0.10$ and 0.15 , with the asymmetry most pronounced at the largest surge amplitude considered. The abrupt loss of power is a result of the rotor stalling at higher surge amplitudes.

Mean $C_{P}$ and $C_{T}$ decreases as the surge amplitude is increased. Table II shows the percentage reduction of the mean $C_{P}$ and $C_{T}$ for various surge amplitudes compared to the fixed device. The reduction in mean thrust and power is due to less optimal rotor operation due to the hydrodynamic effects associated with oscillating inflow.

Fig. 12 (left) shows a sketch of a 2-dimensional aerofoil section on the blade to explain why stall occurs when the turbine experiences surge motion. A fixed turbine rotating at the optimum $\lambda$ maintains an angle of attack $\alpha$ somewhat less than the static stall angle $\alpha_{S}$ (e.g. Fig. 12 right) and the flow remains attached to the blade surface. However, when the same turbine, rotating at constant speed, surges in the upstream direction, $U_{a p}$ increases, 

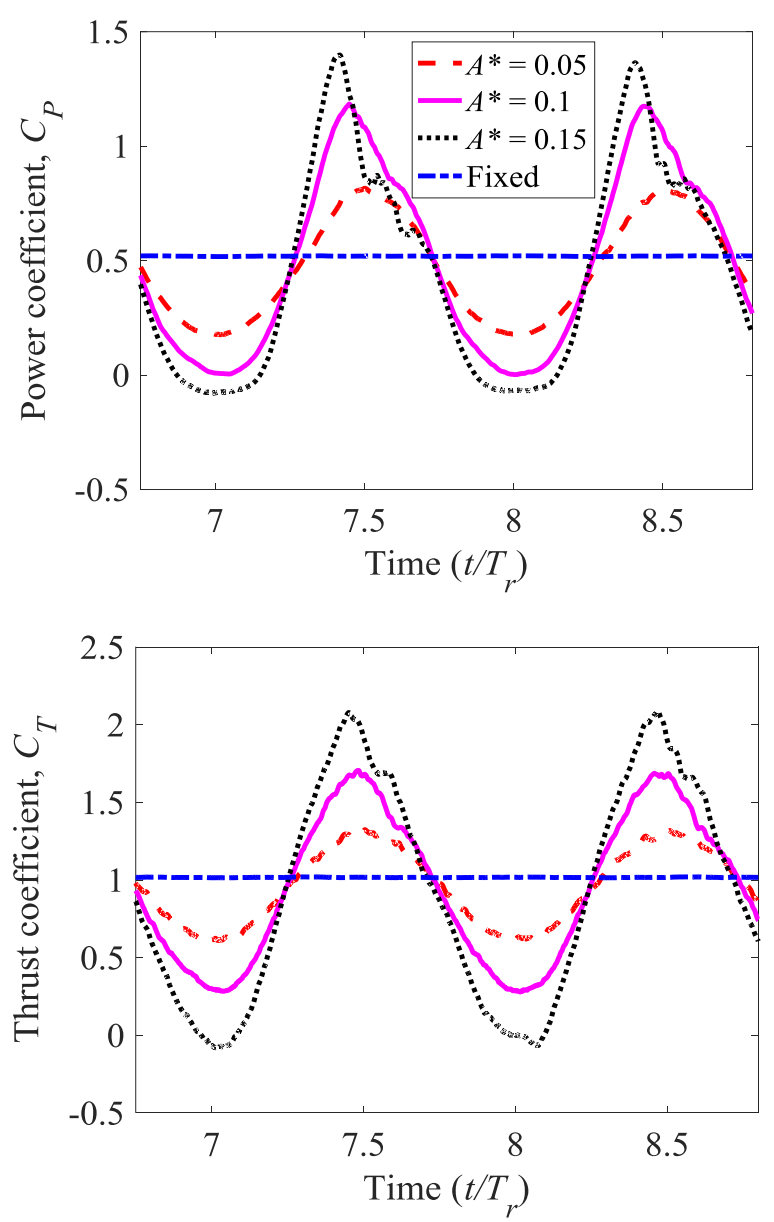

Fig. 11. Time histories of power (top) and thrust (bottom) coefficient for fixed and surging turbine at $A^{*}=0.05,0.10,0.15$ at fixed values of $\lambda=4.4$ and $\omega^{*}=1.0$.

TABLE II

PERCENTAGE DIFFERENCE BETWEEN MEAN POWER AND MEAN THRUST COEFFICIENTS FOR DIFFERENT SURGE AMPLITUDES

\begin{tabular}{lllcc}
\hline$A^{*}$ & $\begin{array}{l}\text { Mean } \\
C_{T}\end{array}$ & $\begin{array}{l}\text { Mean } \\
C_{P}\end{array}$ & $\begin{array}{l}\text { Percentage } \\
\text { reduction } C_{T}(\%)\end{array}$ & $\begin{array}{l}\text { Percentage } \\
\text { reduction } C_{P}(\%)\end{array}$ \\
\hline Fixed & 1.017 & 0.519 & - & - \\
0.05 & 0.960 & 0.467 & 5.60 & 10.02 \\
0.10 & 0.950 & 0.493 & 6.59 & 5.01 \\
0.15 & 0.920 & 0.458 & 9.54 & 11.75 \\
\hline
\end{tabular}

causing $\alpha$ to increase, and possibly exceed, $\alpha_{S}$, causing flow separation to occur along the blade.

Axial and tangential force profiles for the maximum surge amplitude case $\left(A^{*}=0.15\right)$ are presented in Fig. 13 . The largest increases in both axial and tangential force occur at spanwise distances less than $r / R=0.5$ when $U_{a p}$ is maximum, increasing further slightly when the blades experience the largest degree of flow separation at $t / T_{r}=$ 8.53 after maximum $U_{a p}$ is achieved. Axial force outboard of this position is reduced due to the occurrence of flow separation along the blade.

Axial and tangential components of $U_{a p}$ for the surging turbine around a blade cross-section at $r / R=0.7$ are shown in Fig. 14 for the cases of maximum $U_{a p}$ and maximum flow separation. The blade angle of attack is increased significantly compared to the fixed turbine (Figs. 8(c) and (d)), leading in particular to a larger drag force. The presence of separated flow is visible on the

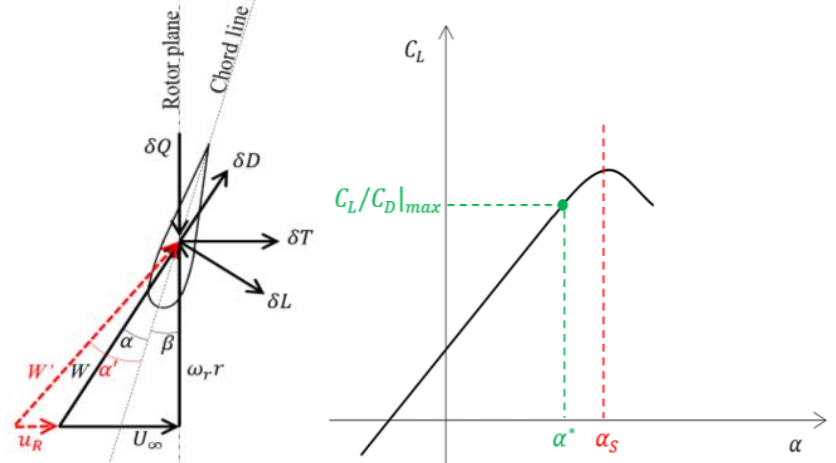

Fig. 12. 2-dimensional sketch (left) of a blade cross-section and a typical lift coefficient over angle of attack curve (right). $u_{R}=\dot{x}(t)=$ surge velocity, $\alpha^{*}=$ design angle of attack, and $\alpha_{S}=$ static stall angle.
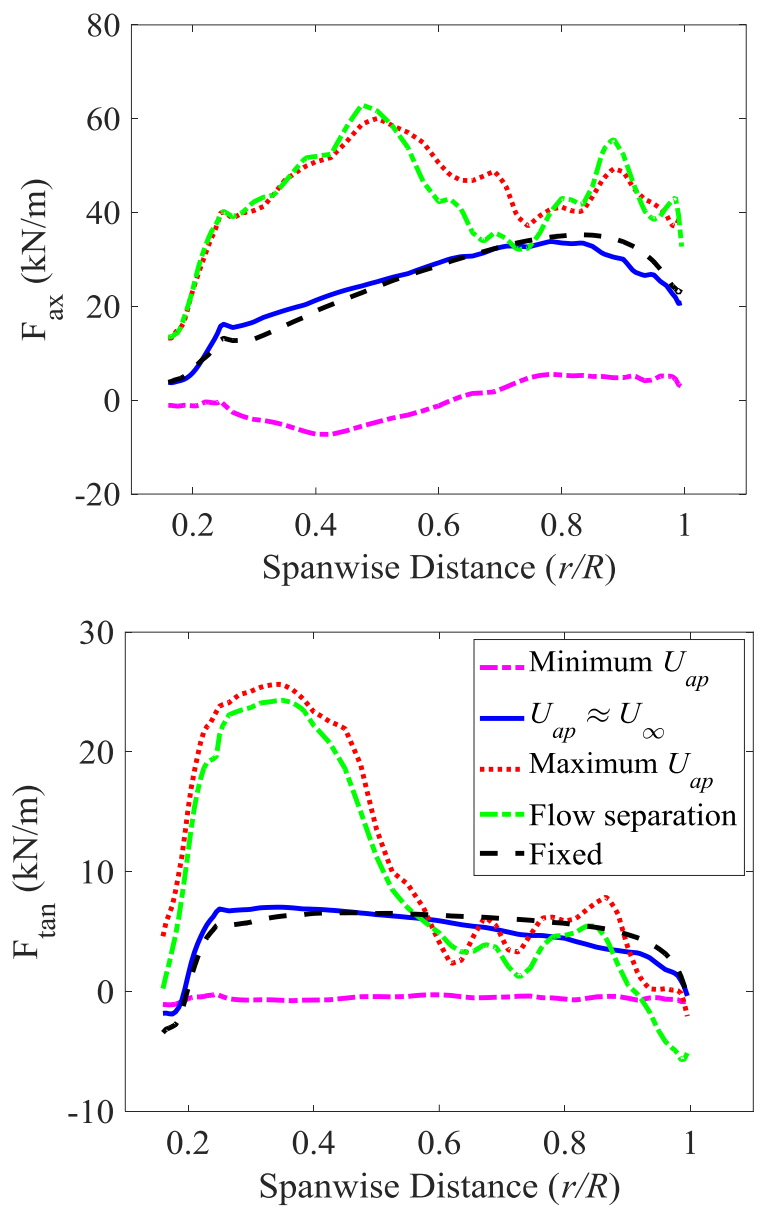

Fig. 13. Axial and tangential force profiles along the blade for fixed and surging turbine $\left(A^{*}=0.15\right)$ corresponding to the minimum $U_{a p}, U_{a p} \approx U_{\infty}$, maximum $U_{a p}$, and when the most significant flow separation occurs.

suction side of the blade. The spanwise variation in $\alpha$ for the fixed and surging turbines is shown in Fig. 15, and shows $\alpha$ is increased most significantly above that for the fixed turbine case when $U_{a p}$ for the surging turbine is maximum. This qualitatively follows the behavior results presented in the previous section, where it was found stall occurred on the blade at large values of $U_{a p}$, which results in large angles of attack on the blade. Increasing the amplitude of oscillation, for a given frequency, results in higher $U_{a p}$ and thus $\alpha>a_{S}$ each cycle, and consequently the effects of stall are more significant for 


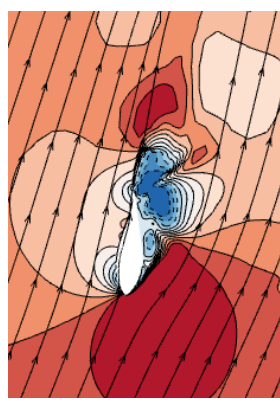

(a) Axial velocity

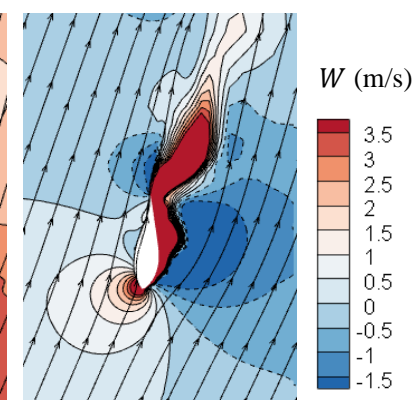

(b) Tangential velocity

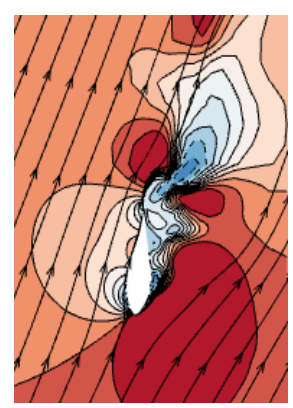

(c) Axial velocity

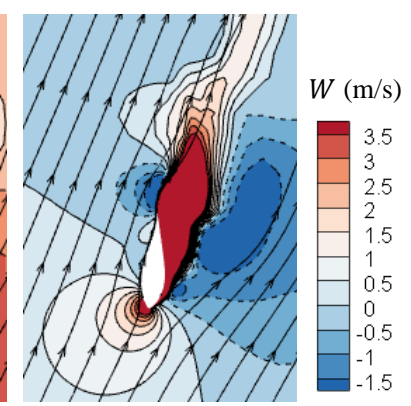

(d) Tangential velocity
Fig. 14. Contour plots of axial and tangential velocities at $r / R=0.7$ for surging turbine when $U_{a p}$ is maximum (a and $\mathrm{b}$, respectively) and when most flow separation occurs (c and $d$, respectively). Streamlines of the resultant velocity $W$ at the blade cross-section are superimposed on the contour plots.

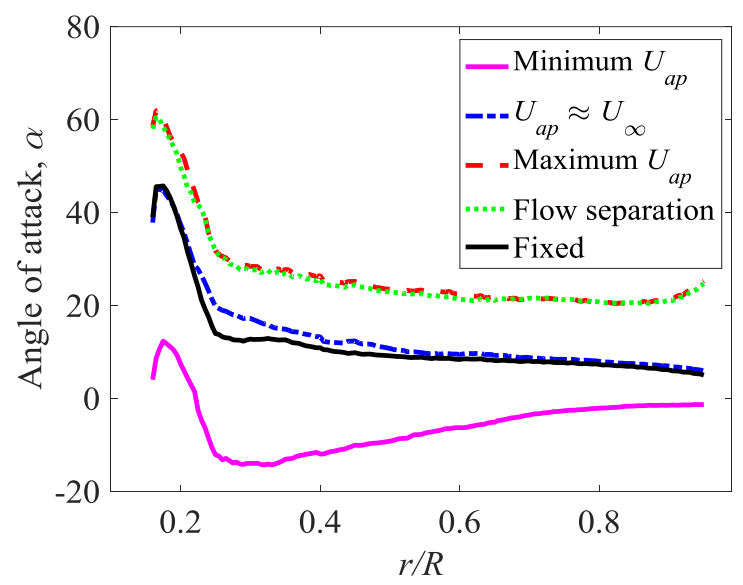

Fig. 15. Angle of attack $\alpha$ profiles along the blade for fixed and surging turbine corresponding to the minimum $U_{a p}, U_{a p} \approx U_{\infty}$, maximum $U_{a p}$, and when the greatest flow separation occurs.

turbines undergoing larger surge oscillations.

Negative values of $C_{P}$ and $C_{T}$ are observed in the maximum surge case when $U_{a p}$ is minimum (Fig. 11), with corresponding negative axial and tangential forces along significant portions of the blade (Fig. 13). As shown in Fig. 16, the low value of $U_{a p}$ means that $\alpha$ for parts of the blade is negative. Recall for these simulations that the rotor rotational speed is held constant. Two situations may arise: a low angle of incidence leading to a change in the drag vector; and larger changes in angle of attack such that lift becomes negative.

In the first case, when $\alpha$ is negative and small in magnitude, the lift may still be positive, defined as the lift vector being on the suction-side of the blade $\left(\delta L_{1}{ }^{\prime}\right.$, Fig.

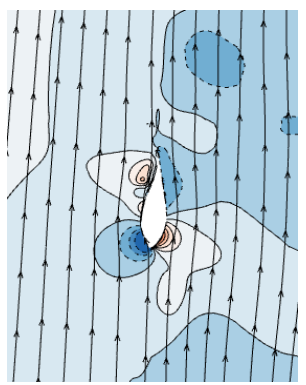

(a) Axial velocity

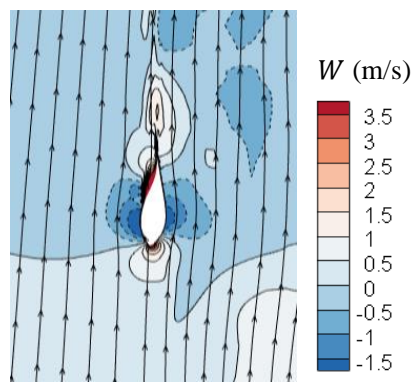

(b) Tangential velocity
Fig. 16. Contour plots of axial (a) and tangential (b) components of $U_{a p}$ at $r / R=0.7$ for a surging turbine for minimum $U_{a p}$. Streamlines of the resultant velocity $W$ at the blade cross-section are. The axial velocity is positive from left to right and the tangential flow is positive going downward.
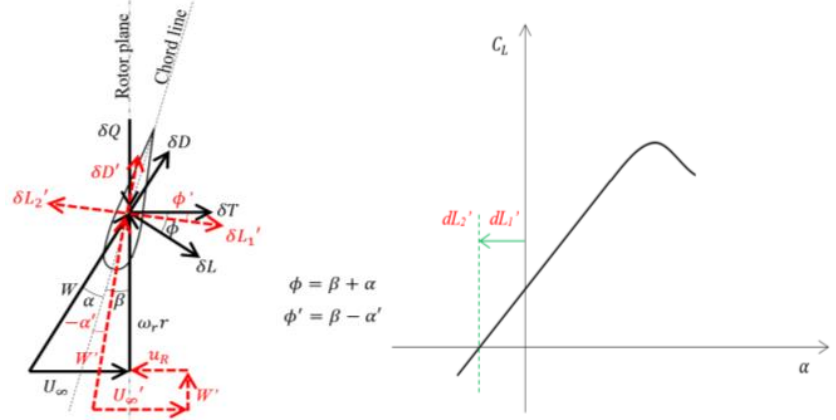

Fig. 17. 2-dimensional sketch (left) of a blade cross-section. Typical lift coefficient over angle of attack curve (right).

17). However, the drag will act at the pressure-side of the blade, decreasing thrust. In the second case, as $\alpha$ reduces further, the lift develops on the pressure side of the blade ( $\delta L_{2}{ }^{\prime}$, Fig. 17$)$, causing the thrust to be negative. In this condition (i.e. thrust and power become negative), the rotor operates more like a propeller than a turbine. As shown in Fig. 15, at minimum $U_{a p}, \alpha$ is negative along much of the blade span. Thus, instead of extracting power from the flow, the rotor requires needs power to continue rotating at the same rotational speed. Consequently, negative $C_{P}$ and $C_{T}$ values may arise during large surge oscillations.

\section{Effect of Surge Frequency on Turbine Performance}

Three cases of normalized surge frequency $\omega^{*}=0.7,1.0$, and 1.3 , were simulated to study the effects of surge frequency on turbine performance. The values of $A^{*}$ and $\lambda$ were kept constant for all three cases (0.1 and 4.4, respectively). The values of $\omega^{*}$ were chosen to be close to the resonant point which lies in a sensible range of wave frequencies, as suggested by [7].

Fig. 18 shows the time histories of $C_{P}$ and $C_{T}$ for three different surge frequencies. As discussed in previous sections, $C_{P}$ and $C_{T}$ are largely in phase with $U_{a p}$ as the power and thrust are proportional to the velocity cubed and velocity squared, respectively. Both the frequency and the amplitude of the fluctuating component of $C_{P}$ and $C_{T}$ increase as the surge frequency increases. Following (4), the apparent velocity experienced by the rotor will 

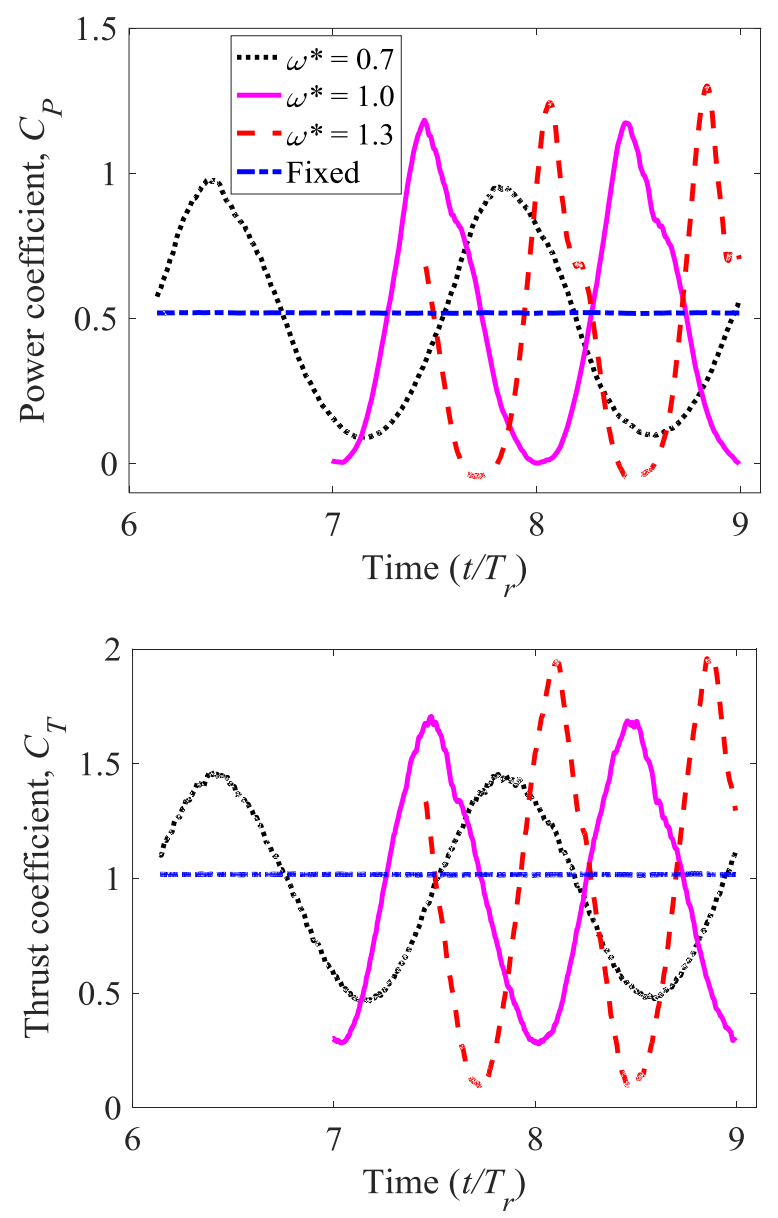

Fig. 18. Time histories of power and thrust coefficient for fixed and surging turbine oscillating at $\omega^{*}=0.7,1.0$, and 1.3. The $A^{*}=0.1$ and $\lambda=4.4$ were kept constant.

increase with $\omega^{*}$ as $u_{R}(t)=\dot{x}(t)=A_{0} \omega_{0} \cos \left(\omega_{0} t\right)$. Higher oscillation frequencies will cause more fatigue damage to the rotor compared to an increase in surge amplitude leading to the same amplitude of $U_{a p}$ alone, as the damage is sustained at a higher rate. In the open sea, it is likely than an increase in both surge amplitude and frequency will occur (relative to the non-oscillating case), and this will be an important factor in determining the fatigue life of floating tidal turbine systems.

Asymmetries in thrust and power at the peak of each cycle occur in higher $\omega^{*}$ cases, indicating that flow separation occurs when the surge frequency is increased. The axial and tangential force profiles for the maximum surge frequency case are presented in Fig. 19. As in the maximum surge amplitude case presented previously, there is a significant change in the force profile from the fixed turbine case when flow separation is most significant (from $r / R=0.5$ to the blade tip), showing the rotor goes into stall.

It is observed in Fig. 18 that, for the maximum surge frequency at minimum $U_{a p}, C_{P}$ is negative while $C_{T}$ remains positive. The spanwise variation in tangential force (Fig. 19) is small and negative in this case, with the rotor torque dominated by the drag component due to the small but positive flow incidence angle. Consequently, rotor power is negative.
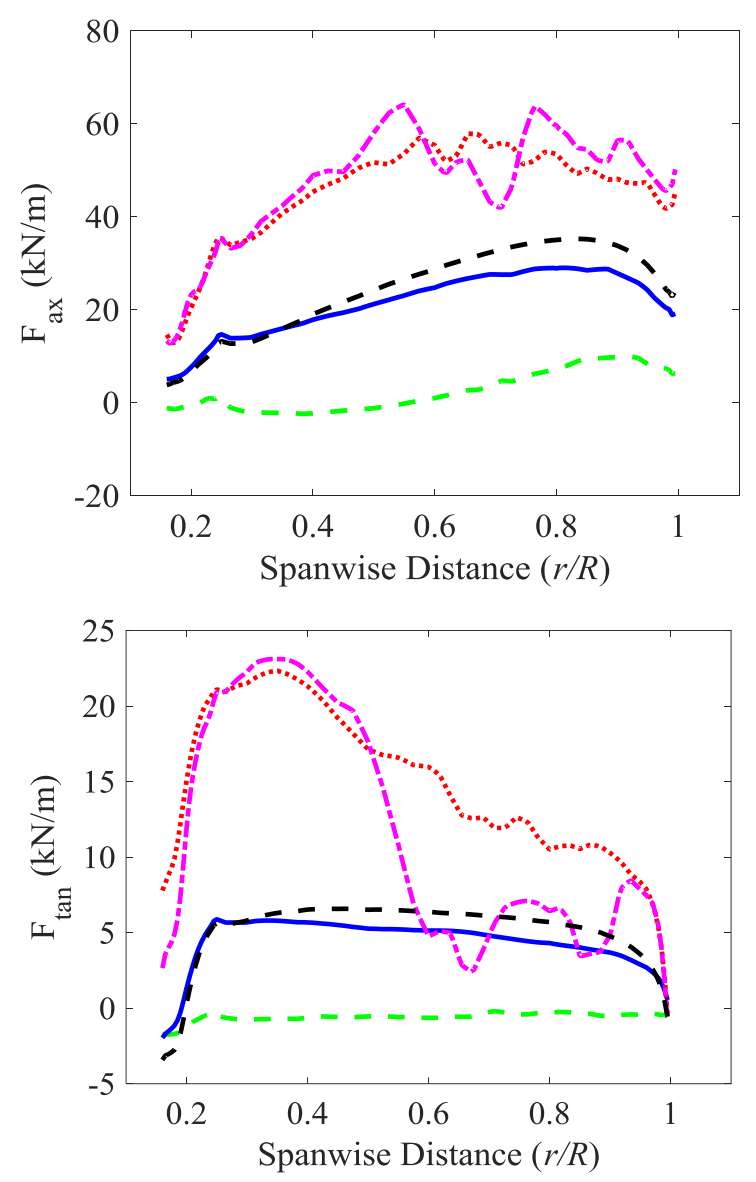

- $=$ Minimum $U_{a p} \quad-U_{a p} \approx U_{\infty} \quad \ldots . .$. Maximum $U_{a p}$
-=-Flow separation $\quad-$-Fixed

Fig. 19. Axial (top) and tangential (bottom) force profiles along the blade for fixed and surging turbine at minimum $U_{a p}, U_{a p} \approx U_{\infty}$, maximum $U_{a p}$, and when the most flow separation occurs. All results are for the maximum surge frequency case $\left(\omega^{*}=1.3\right)$.

Negative values of axial force per unit span are restricted to inboard sections between $r / R=0.3$ to 0.5 at minimum $U_{a p}$. The thrust is largely a function of sectional lift on the outboard sections where the flow incidence angle is small, and thus the minimum $C_{T}$ remains positive at the highest oscillation frequency considered.

Table III shows the percentage change in the mean power and mean thrust coefficients for the three different surge frequencies when compared to the non-oscillating (fixed) case. References [17] and [18] conducted numerical analyses on a horizontal axis floating offshore wind turbine oscillating in surge motion using a free vortex method and a lifting surface method, respectively. It was found that mean power and thrust increase as the surge amplitude and frequency is increased. Reference [2] conducted a similar CFD study on a HATT. They found no change in the mean power and thrust of a surging turbine compared to a fixed turbine when the surge amplitude and frequency is increased. In the present study, it was found that the mean $C_{T}$ reduced slightly when surge amplitude and frequency is increased (Tables II and III), although mean $C_{P}$ was found to increase at resonant amplitude and frequency, although was still less 
TABLE III

PERCENTAGE DIFFERENCE BETWEEN MEAN POWER AND MEAN THRUST COEFFICIENTS FOR DIFFERENT SURGE FREQUENCIES

\begin{tabular}{llccc}
\hline$\omega^{*}$ & $\begin{array}{l}\text { Mean } \\
C_{T}\end{array}$ & $\begin{array}{l}\text { Mean } \\
C_{P}\end{array}$ & $\begin{array}{l}\text { Percentage } \\
\text { reduction } \\
C_{T}(\%)\end{array}$ & $\begin{array}{l}\text { Percentage } \\
\text { reduction } \\
C_{P}(\%)\end{array}$ \\
\hline Fixed & 1.017 & 0.519 & - & - \\
0.7 & 0.959 & 0.484 & 5.70 & 6.74 \\
1.0 & 0.950 & 0.493 & 6.59 & 5.01 \\
1.3 & 0.927 & 0.438 & 8.85 & 15.61 \\
\hline
\end{tabular}

than that for a fixed turbine. This is likely due to differences in the blockage and the rotor design in the different studies, but flow parameters and the change in the reference frame may also play a role. In all surging turbine cases, it was found that the mean $C_{P}$ and $C_{T}$ were less than the fixed turbine case. This is a consequence of the surging rotor spending significant fractions of each cycle operating away from the turbine's optimal operating conditions.

\section{CONCLUSIONS}

CFD simulations were conducted to study the effects of surge oscillation on the hydrodynamic performance of a floating horizontal axis tidal turbine. In all cases, turbine oscillation was found to reduce the mean thrust and power coefficients relative to the case of a non-oscillating turbine.

As the amplitude and frequency of the surge oscillations increase, the unsteady loading on the rotor also increases. Flow separation is seen to occur on the blades at maximum $U_{a p}$ (turbine advancing into the flow), where significant portions of the rotor blade are stalled. During stall, the thrust amplitude increases and may fluctuate at sufficiently high surge amplitudes and frequencies, which will contribute to fatigue damage of the rotor.

The rotational speed of the turbine was held constant throughout the surge oscillation cycle. Consequently, when the rotor retreats the apparent velocity $U_{a p}$ was sufficiently small in the largest amplitude and frequency cases to cause the sectional lift and drag forces to rotate from the suction to the pressure surface of the blade. This resulted in the rotor producing negative instantaneous thrust and power coefficients, as the rotor operated like a propeller rather than a turbine under such conditions.

\section{ACKNOWLEDGEMENT}

M. H. B. Osman thanks Majlis Amanah Rakyat Malaysia (MARA) and Universiti Teknologi Malaysia (UTM) for financial support, and Oxford University Advanced Research Computing (ARCUS) for providing high performance computing system services.

\section{REFERENCES}

[1] K. Wang, K. Sun, Q. Sheng, L. Zhang, S. Wang, "The effects of yawing motion with different frequencies on the hydrodynamic performance of floating vertical-axis tidal current turbines," Journal of Applied Ocean Research, vol. 59, pp. 224-235, 2016a.

[2] L. Zhang, S. Wang, Q. Sheng, F. Jing, Y. Ma, “The effects of surge motion of the floating platform on hydrodynamics performance of horizontal-axis tidal current turbine," Journal of Renewable Energy, vol. 74, pp. 796-802, 2015.

[3] S. Wang, K. Sun, J. Zhang, L. Zhang, "The effects of roll motion of the floating platform on hydrodynamics performance of horizontal-axis tidal current turbine," Journal of marine science and technology, 2016.

[4] F. Jing, G. Xiao, N. Mehmood, L. Zhang, "Optimal selection of floating platform for tidal current power station," Research Journal of Applied Sciences, Engineering and Technology, vol. 6, pp. 1116-1121, 2013.

[5] F. R. Menter, "Two-equation eddy-viscosity turbulence models for engineering applications," Journal of American Institute of Aeronautics and Astronautics, vol. 32, no. 8, 1994.

[6] A. Wimshurst, R. H. J. Willden, "Computational analysis of blockage designed tidal turbine rotors," Progress in Renewable Energies Offshore, ISBN 978-1-138-62627-0, 2016.

[7] J. Schluntz, R. H. J. Willden, "The effect of blockage on tidal turbine rotor design and performance," Journal of Renewable Energy, vol. 81, pp. 432-441, 2015.

[8] C. F. Fleming, "Tidal turbine performance in the offshore environment," Ph.D. dissertation, St. Catherine's College, University of Oxford, 2014.

[9] I. Afgan, J. McNaughton, S. Rolfo, D. D. Apsley, T. Stallard, P. Stansby, "Turbulent flow and loading on a tidal stream turbine by LES and RANS," International Journal of Heat and Fluid Flow, vol. 43, pp. 96-108, 2013.

[10] H. J. Aguerre, S. M. Damián, J. M. Gimenez, N. M. Nigro, "Engine simulation using layering and sliding interfaces mesh dynamics technologies," Journal of Computational Mechanics, vol. 33 pp. 27-49, 2014.

[11] J. Camargo, O. Lopez, N. Ochoa-Lleras, “A computational tool for unsteady aerodynamic flow simulations coupled with rigid body dynamics and control," in American Institute of Aeronautics and Astronautics Applied Aerodynamics Conference, 2012, vol. 30.

[12] L. Li, S. J. Sherwin, P. W. Bearman, "A moving frame of reference algorithm for fluid-structure interaction of rotating and translating bodies," International Journal for Numerical Methods in Fluids, vol. 38 pp. 187-206, 2002.

[13] J. R. Meneghini, P. W. Bearman, "Numerical simulation of high amplitude oscillatory flow about a circular cylinder," Journal of fluids and structures, vol. 9 pp. 435-455, 1995.

[14] S. H. Madani, J. Wissink, H. Bahai, "Modelling inertial forces on cylinders in cross flow using moving frame of reference," in Proceedings of the 24th UK Conference of the Association for Computational Mechanics in Engineering, 2016.

[15] D. Micallef and T. Sant, "Loading effects on floating offshore horizontal axis wind turbines in surge motion," Journal of Renewable Energy, vol. 83 pp. 737-748, 2015.

[16] J. B. de Vaal, M. O. L. Hansen, T. Moan, "Effect of wind turbine surge motion on rotor thrust and induced velocity," Journal of Wind Energy, vol. 17 pp. 105-121, 2014.

[17] B. Wen, X. Tian, X. Dong, Z. Peng, W. Zhang, “ Influences of surge motion on the power and thrust characteristics of an offshore floating wind turbine," Journal of Energy, vol. $141 \mathrm{pp}$. 2054-2068, 2017.

[18] X. Shen, J. Chen, P. Hu, X. Zhu, Z. Du, "Study of the unsteady aerodynamics of floating wind turbines," Journal of Energy, vol. 145 pp. 793-809, 2018. 